\title{
A QUESTION ON INVARIANT SUBSPACES OF BERGMAN SPACES
}

\author{
HARI BERCOVICI
}

(Communicated by John B. Conway)

\begin{abstract}
We answer a question raised by Axler and Bourdon [1] concerning the classification of finite-codimensional invariant subspaces of Bergman spaces.
\end{abstract}

Let $\Omega$ be a bounded domain in $\mathbf{C}^{n}, 1 \leq p<\infty$, and denote by $A^{p}(\Omega)$ the Bergman space consisting of all holomorphic functions in $\Omega$, whose $p$ th power has finite Lebesgue integral. A subspace $M \subset A^{p}(\Omega)$ is said to be invariant if $T_{j} M \subset$ $M, 1 \leq j \leq n$, where $T_{j}$ denotes multiplication by the $j$ th coordinate function $z_{j}$. Axler and Bourdon give in [1] a characterization of the finite-codimensional invariant subspaces of $A^{p}(\Omega)$ for sufficiently nice domains $\Omega$. More precisely, they show that given such a subspace $M$, there exist linear partial differential operators $L_{1}, L_{2}, \ldots, L_{q}$ with constant coefficients, and points $\lambda^{(1)}, \lambda^{(2)}, \ldots, \lambda^{(q)} \in \Omega$, such that

$$
M=\left\{f \in A^{p}(\Omega):\left(L_{j} f\right)\left(\lambda^{(j)}\right)=0, j=1,2, \ldots, q\right\} .
$$

They raised the following natural question. Given linear partial differential operators $L_{1}, L_{2}, \ldots, L_{q}$ with constant coefficients, and points $\lambda^{(1)}, \lambda^{(2)}, \ldots, \lambda^{(q)} \in \Omega$, under what conditions is the space $M$ defined by (1) invariant? Our purpose in this note is to provide such necessary and sufficient conditions. To formulate our result we need some notation. For a polynomial $P\left(\xi_{1}, \xi_{2}, \ldots, \xi_{n}\right) \in \mathbf{C}\left[\xi_{1}, \xi_{2}, \ldots, \xi_{n}\right]$, we denote by $P(D)$ the corresponding linear partial differential operator. Thus, if $P(\xi)=\xi_{j}$, then $P(D)=\partial / \partial z_{j}, 1 \leq j \leq n$. Given $P \in \mathbf{C}\left[\xi_{1}, \xi_{2}, \ldots, \xi_{n}\right]$ and $\lambda=\left(\lambda_{1}, \lambda_{2}, \ldots, \lambda_{n}\right) \in \Omega$, we define a functional $\varphi(P, \lambda)$ in the dual $\left(A^{p}(\Omega)\right)^{\prime}$ of $A^{p}(\Omega)$ by

$$
\varphi(P, \lambda)(f)=(P(D) f)(\lambda), \quad f \in A^{p}(\Omega) .
$$

Observe that the Leibniz formula (cf. formula (1.4.12) in [2]) yields immediately the equality

$$
P(D)\left(T_{j} f\right)=T_{j} P(D)+\frac{\partial P}{\partial \xi_{j}}(D) f, \quad 1 \leq j \leq n
$$

and hence

$$
T_{j}^{\prime} \varphi(P, \lambda)=\lambda_{j} \varphi(P, \lambda)+\varphi\left(\frac{\partial P}{\partial \xi_{j}}, \lambda\right), \quad 1 \leq j \leq n,
$$

where $T_{j}^{\prime}$ denotes the dual operator of $T_{j}$, acting on $\left(A^{p}(\Omega)\right)^{\prime}$.

Received by the editors March 4, 1987 and, in revised form, March 23, 1987.

1980 Mathematics Subject Classification (1985 Revision). Primary 47A15, 47B38. 
Proposition. Let $\Lambda \subset \Omega$ be a finite set, and for each $\lambda \in \Lambda$ let $\mathscr{F}$ be a finite subset of $\mathbf{C}\left[\xi_{1}, \xi_{2}, \ldots, \xi_{n}\right]$. The subspace $M=\left\{f \in A^{p}(\Omega):(P(D) f)(\lambda)=0\right.$, $\left.P \in \mathscr{F}_{\lambda}, \lambda \in \Lambda\right\}$ is invariant if and only if for each $\lambda \in \Lambda$, the linear space generated by $\mathscr{F}_{\lambda}$ is invariant under $\partial / \partial \xi_{j}, 1 \leq j \leq n$.

ProOF. The space $M^{\perp}=\left\{\varphi \in\left(A^{p}(\Omega)\right)^{\prime}: \varphi \mid M=0\right\}$ coincides with the linear space generated by $\left\{\varphi(P, \lambda): \lambda \in \Lambda, P \in \mathscr{F}_{\lambda}\right\}$. Assume first that the linear space generated by $\mathscr{F}_{\lambda}$ is invariant under $\partial / \partial \xi_{j}$ for all $\lambda \in \Lambda$. Formula (2) shows then that $M^{\perp}$ is invariant under $T_{j}^{\prime}$, and hence $M$ is invariant under $T_{j}, 1 \leq j \leq n$. Conversely, assume that $M$ is invariant under $T_{j}$. Then $M^{\perp}$ is invariant under $T_{j}^{\prime}$, $1 \leq j \leq n$. Again, formula (2) shows that in this case $\varphi\left(\partial P / \partial \xi_{j}, \lambda\right)$ must belong to $M^{\perp}$ whenever $\lambda \in \Lambda, P \in \mathscr{F}_{\lambda}$, and $1 \leq j \leq n$. Thus, for fixed $\lambda, P$ and $j$, there exist scalars $\alpha_{\mu, Q}$ such that

$$
\varphi\left(\frac{\partial P}{\partial \xi_{j}}, \lambda\right)=\sum_{\mu \in \Lambda} \sum_{Q \in \mathscr{F}_{\mu}} \alpha_{\mu, Q} \varphi(Q, \mu)
$$

Since there are functions in $A^{p}(\Omega)$ that have zeros of arbitrarily high order at all points $\mu \in \Lambda \backslash\{\lambda\}$, and with prescribed partial derivatives up to a certain order at $\lambda$, the last equality implies that $\partial P / \partial \xi_{j}=\sum_{Q \in \mathscr{F}_{\lambda}} \alpha_{\lambda, Q} Q$. Thus $\partial P / \partial \xi_{j}$ belongs to the linear space generated by $\mathscr{F}_{\lambda}$. Since $j, P$, and $\lambda$ are arbitrary, the proposition follows.

The criterion given in the above proposition is quite easy to apply. The reader will be able to verify at a glance that, for fixed $\lambda \in \Omega$ and $c \in \mathbf{C}$, the finite codimensional subspace

$$
\left\{f \in A^{p}(\Omega): f(\lambda)=\frac{\partial f}{\partial z_{1}}(\lambda)+\frac{\partial f}{\partial z_{2}}(\lambda)=c \frac{\partial^{2} f}{\partial z_{1} \mid \partial z_{2}}(\lambda)+\frac{\partial^{2} f}{\partial z_{1}^{2}}(\lambda)+\frac{\partial^{2} f}{\partial z_{2}^{2}}(\lambda)=0\right\}
$$

is invariant if and only if $c=2$. This example was suggested by the referee.

\section{REFERENCES}

1. S. Axler and P. Bourdon, Finite codimensional invariant subspaces of Bergman spaces, preprint.

2. L. Hörmander, Linear partial differential operators, Springer-Verlag, Berlin and New York, 1963.

DEPARTMENT OF MATHEMATICS, INDiANA UNIVERSity, BloOMington, INDiANA 47405 